Journal of University of Shanghai for Science and Technology

\title{
Periodic variations of the geomagnetic activity indices Ap and Kp
}

\author{
D.O.I - 10.51201/Jusst12634 \\ http://doi.org/10.51201/Jusst12634
}

\author{
A. C. Pandey ${ }^{1}$, Sham Singh ${ }^{2 *}$, Dinesh Kumar Pathak ${ }^{3}$, Archana Shukla ${ }^{3}$, Amita Rani ${ }^{4}$ \\ and A.P. Mishra ${ }^{3}$ \\ ${ }^{1}$ Department of Physics, Govt. New Science College Rewa (M. P.) India \\ ${ }^{2}$ Department of Applied Sciences, Chandigarh Engineering College, Mohali Punjab 140307 India \\ ${ }^{3}$ Department of Physics, A. P. S. University, Rewa (M. P.) 486003 India \\ ${ }^{4}$ Department of Physics, Barkatullah University, Bhopal 462026 India
}

\begin{abstract}
Yearly averages of geomagnetic activity indices Kp and Ap for the years 1984 to 2018 be compared to the relevant averages of $\mathrm{VxBs}$, where $\mathrm{V}$ is the solar wind velocity and $\mathrm{Bs}$ is the southward interplanetary magnetic field (IMF) component. The correlation of both quantities is known to be rather good. Comparing the averages of Ap and Kp with V and Bs separately. We found that, during the declining phase of solar cycle, $\mathrm{V}$ and during the ascending phase Bs have more influence on Ap and Kp indices. According to this observation the 27 days and semiannual, Ap and Kp variations be analysed discretely for years after and before sunspot minima. The time intervals prior to sunspot minima with a significant 27-day recurrent period of the IMF structure and those intervals after sunspot minima with a significant 28 to 28.5 day recurrent phase of the structure be used. The averaged spectra of the two Ap and Kp data sets obviously show a period of 27 days before and a period of 28 to 29 days after sunspot minimum.
\end{abstract}

Key words: Interplanetary Magnetic field, Solar wind plasma and magnetosphere Interaction.

\section{Introduction}

The interaction of the solar wind is the source of geomagnetic activity. While the first in situ interpretation of solar wind, frequent studies have been carried out to find quantitative 
associations among the solar parameters and its variability with geomagnetic activity. Solar wind speed V and the southward-component Bs of the interplanetary magnetic field IMF be realized to the essential quantity causes geomagnetic activity (Arnoldy 1971; Berthelier 1976). Sources of soaring solar wind velocity connected with southward IMF which produce increased geomagnetic activity at the earth, originate in speedy coronal mass ejections (CMEs), interaction regions and coronal holes among slow and speedy solar wind (Boller and Stolov 1970; Cortie 1912; Crooker and Cliver 1994). Studies relating to solar wind uniqueness with the geomagnetic activity can expose the physics of the interaction of solar wind plasma and magnetospheric.

Most observations propose with the intention of the solar wind plasma be transfer to the magnetosphere by reconnection progression at the dayside magnetopause and oppositely directed northward magnetospheric and southward IMF magnetic field lines merge (Dungey 1961). Theoretical models of the merging process have been described (Singh and Mishra 2019) however, not all details of the physics are yet understood (Dungey 1994) and still other energy transfer mechanisms such as viscous interaction and Kelvin-Helmholtz wave instabilities at the flanks the magnetosphere be discussed (Fraser-Smith 1972).

The problem of the physics prevailing the relation to the solar wind speed with the geomagnetic activity can be investigated by using synoptic observations of single events or by statistical analysis of time-series of the crucial quantities. Here, we pursue the latter method as the study of the periodic variation in the sun-earth-system, the 11-years sunspot cycle period, 27-day period, the semiannual and in addition to the diurnal variation. We show that in all these predictions and not just the field line merger or reconnection development is visible and also especially at exacting phases of the solar cycle that other mechanisms may be effective. 


\section{Results and discussion}

The periodic variations of the solar cycle and Ap index to study the long period variations, we have to use the Ap indices as every day averages of ap. The 11-year periodic of the geomagnetic activity runs about parallel toward the cycle of the sunspot number R; though, it is recognized that here are significant differences during the two cycles as geomagnetic activity has two peaks, one near the sunspot maximum and other in the declining phase of the sunspots. These two peaks have been seen to be mostly significant in the distribution of geomagnetic storms (Singh et al 2012). In commonly, the activity minimum appears 2 or 3 years behind the sunspot minimum.

Spectral examination of $\mathrm{R}$ and Ap expose a prominent peak within the spectral influence of $\mathrm{R}$ at the periods of 10.2-10.4 years, whereas, the Ap refusal analogous maximum (Gonzalez 1987; Gonzalez et al 1990). The Fourier spectral study of the time-intervals of Ap index and R 1933 1993 give no latest results during the 11-year spectral series and therefore, not shown in Fig. 1 yearly average of some quantities, regarding the following considerations, are shown on behalf of the years 1968 to $1984 / 1986$. In the upper part, Ap and v2.Bs=105 have comparable scales, where v2 and Bs are, respectively, the yearly average of the squares of hourly values of solar wind velocity $\mathrm{V}$ in $\mathrm{km} / \mathrm{s}$, and southward component $\mathrm{Bs}$ of IMF in $\mathrm{nT}$ and in solar magnetospheric coordinates.

The hourly average values for the solar and interplanetary data be taken from the "OMNI data base compiled by (Gonzalez et al 1993, Green1984) and are available on CD-ROM of the national geophysical data Center, (NOAA/NESDIS/NGDC) Boulder, USA". The yearly Bs values are computed from the hourly IMF-Bz values by setting positive (northward directed) hourly Bz averages equal to zero. In the lower part of Fig. 1 the respective yearly averages of $\mathrm{m} 2$ and Bs are shown separately. In the upper part there is a good correlation of the two curves 
(correlation coefficient $r=0.92$ ). This is expected, since most correlative studies resulted in best correlation between geomagnetic activity and m2Bs (Crooker and Sicoe 1986). The lower part of Fig. 1, however, shows a variable influence of $\mathrm{v}^{2}$ and Bs on the activity.

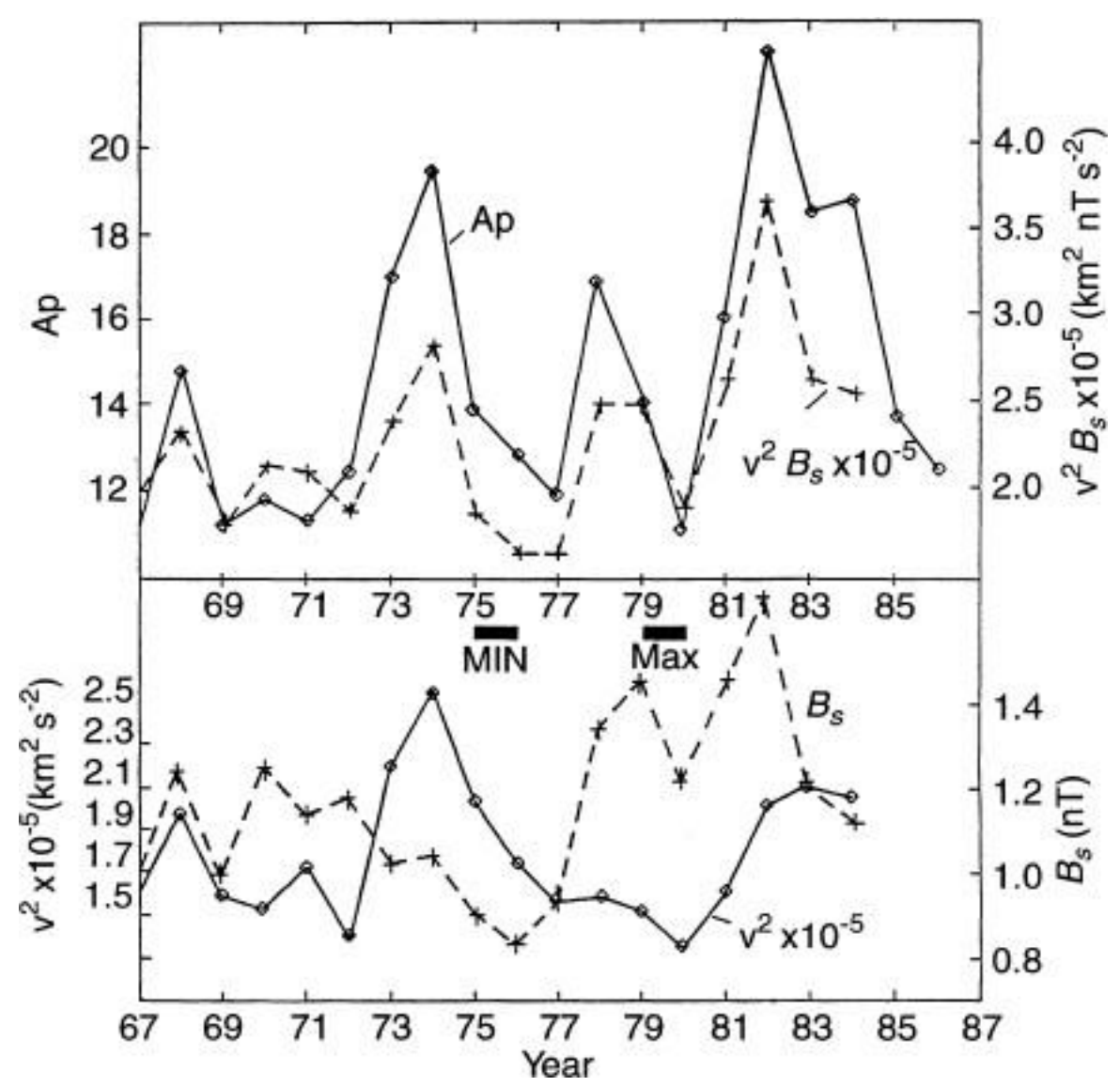

Fig. 1. Yearly averages of Ap for the years 1967-1984 (to 1986 for Ap) upper part, and yearly averages of the southward IMF component Bs and $\mathrm{v}^{2}$; maximum and minimum years of sunspot cycle 21 are indicated

The course of the sunspot cycle; for the period of declining phase in the years 1974-1976 increasing v2 averages result in increased activity, whereas in the ascending phase (1976-1979) the increase in Bs causes the activity increase. It seems that the variables of solar sources be responsible intended for the varying influence of $\mathrm{V}$ and $\mathrm{Bs}$ on the geomagnetic activity. The semiannual variation observation discussed in (Fig.1) aggravated us to examine (in addition to the 11-year solar cycle period) the most prominent periodic variation of the semiannual wave in 
the geomagnetic activity for the time-intervals through high solar wind plasma or with a strong IMF-southwards component.

Although the semiannual activity variation with the maxima about the equinoxes and minima near the solstices, have been recognized for a long time the analysis be still controversial. There are three mechanisms to clarify the semiannual variation. Firstly, there be axial assumption, which connects the maxima of solar activity with the maximal heliographic latitude of the earth $\left(+7.25^{\circ}\right.$ on 6 September) $7.25^{\circ}$ on 5 March). This occurs when the position of the earth is favorable for the active solar regions, while it is recognized that near the solar equator the activity is minimal (Hirshberg and Colburn 1969) The second mechanism based lying on the equinoctial guess (Hoeksema et al 1983; King 1977) suggesting that the interaction among the solar wind speed and magnetosphere is maximum when the direction between solar wind and the Earth's dipole is greatest on the equinoxes (21 March \& 23 September). The third mechanism be based lying on the reconnection model of the interplanetary and the geomagnetic field lines and take into account the southward components of the IMF with respect to the Earth's magnetic field direction (King 1986). This model predicts, on average, maximal southward Bs fields and corresponding maximal geomagnetic activity on 5 April and 5 October. There have been several investigations in current time to choose which models really cause the semiannual wave and if some mechanisms are effective, which of them be predominant (Lindsay et al 1995; McIntosh1959; Murayama1974; Petschek1964; Russell 1989).

Generally, during these studies the phases of activity maxima be analyzed, since the maxima predicted with the different models be not very secluded from each-other and because here are statistical variation during the activity time series. However, it was not clear which of the mechanisms are the mainly appropriate. The maxima are found to become visible a few days 
behind the equinoxes (25-28 March and 27-30 Sept., depending lying on the activity time series type of analysis) therefore the equinoctial mechanism be often favored. However, the studies cited show that at least a crucial part of the semiannual deviation may be explicated by the Russell-McPherron model. Whereas the Russell-McPherron model is the foundation on the southward component of IMF, the two other mechanisms be more dependent lying on the solar wind speed, which increases through the solar latitude or when increased, may perhaps generate the Kelvin-Helm- holtz instability at the of magnetosphere (Schreiber 1998).

Systematic phase shift depending on the classes of V or Bz. The 27-day variation investigating the geomagnetic activity variations within the region around the solar revolving period of approximately 27 days, one obtains spectral amplitudes with varying peaks depending lying on the length of the time-cycle and the method of analysis (Sargent 1986; Singh and Mishra 2015)

. Our fourier spectral amplitude analysis for periods 30-23 days of Ap indices 1933-1993 is shown in the Fig. 2 together with that of the same but shorter time-series 1933-1983. The moderately complex spectrum shows numerous different peaks depending lying on the length of time- cycles. The four major distinct peaks at the period of 29.4, 28.9, 27.6 and 27.2 days for the Ap time-interval 1933-1983 only the 27.2 days period is found the four greatest amplitudes at the periods of 27.2, 26.7, 25.8 and 24.0 days for the 1933-1993 interval. The respective amplitudes, and several more, exceed the rms error of 0.2 Ap units; the mean spectral amplitude in that period range is 0.35 Ap units. 
Table 1. Amplitudes and phases of the semiannual wave of $a_{p}$ for different solar wind speed $v$ and IMF Bz intervals

\begin{tabular}{lrrrlll}
\hline $\begin{array}{l}\text { v interval } \\
\text { Km/s }\end{array}$ & \multicolumn{2}{c}{$\begin{array}{l}\text { B interval } \\
\text { nT }\end{array}$} & $\begin{array}{l}\text { Amplitude } \\
\text { ap-units }\end{array}$ & $\begin{array}{l}\text { Date of } \\
\text { Max. }\end{array}$ & Remarks \\
\hline 380 & 430 & -3.0 & -2.0 & 1.7 & 1 April & Expected \\
380 & 430 & 2.0 & 3.0 & 0.7 & 5 April & Unexpected \\
430 & 450 & -1.5 & -1.0 & 0.7 & 1 April & Expected \\
430 & 450 & 1.0 & 1.5 & 0.7 & 14 March & Expected \\
450 & 500 & -1.5 & -1.0 & 1.7 & 6 April & Expected \\
450 & 500 & 1.0 & 1.5 & 1.5 & 2 April & Unexpected \\
500 & 600 & -1.5 & -1.0 & 1.8 & 12 April & Unexpected \\
500 & 600 & 1.0 & 1.4 & 1.4 & & Unexpected \\
\hline
\end{tabular}

The rather spacious nature of Ap index, Fourier spectrum around the 27-day period is due to nonpersistent periodicities of the geomagnetic activity to generating solar sources, for instance the development of coronal holes and their disappearance, whereas new holes appear at other solar longitudes. Therefore Fourier spectral study is not the appropriate method to analyze the 27-day period of the longer Ap time-series. However, the power spectral analysis as applied by (Schreiber 1981) also resulted in rather complex spectra with several significant peaks, of which the greatest near 27.2 days and that near 29.4 days are also seen in our Fourier spectra.

In contrast, the rotation period of solar sources is known to vary between 26 and 29 days depending of the phase of a solar cycle. Therefore, to investigate the 27 days variation in detail, one should split up the data into shorter time-intervals where perseverance may be expected. First attempts to separate longer (28-29 days) and shorter (26-27 days) solar rotation periods during, the solar cycles, investigating Ap in the 26-29 day period interval for different phases of the solar cycle or for different ranges of sunspot numbers, gave the same unclear results, as seen in spectra of Fig. 2. 
Finally, a discriminating feature that splits the data was found in the varying recurrence period of the sector structure of the interplanetary magnetic field with IMF directions towards and away from the sun, which in reality the wavy structure of the heliospheric current-sheet (Svalgaard 1972; Svalgaard and Wilcox 1975). The recurrence period of this sector structure is known to react on the global scale the average solar rotation period. It also has been shown that a basic variability of the sector structure becomes apparent with characteristic times on the order of the minority " (Tsurutani et al 1995) and the fundamental period changes from 28 to 27 days according the phase of solar cycle. For our analysis we used the IMF polarity inferred from the daily variation of ap, which was found to give reason- able results for statistical purposes Vasilyunas 1997).

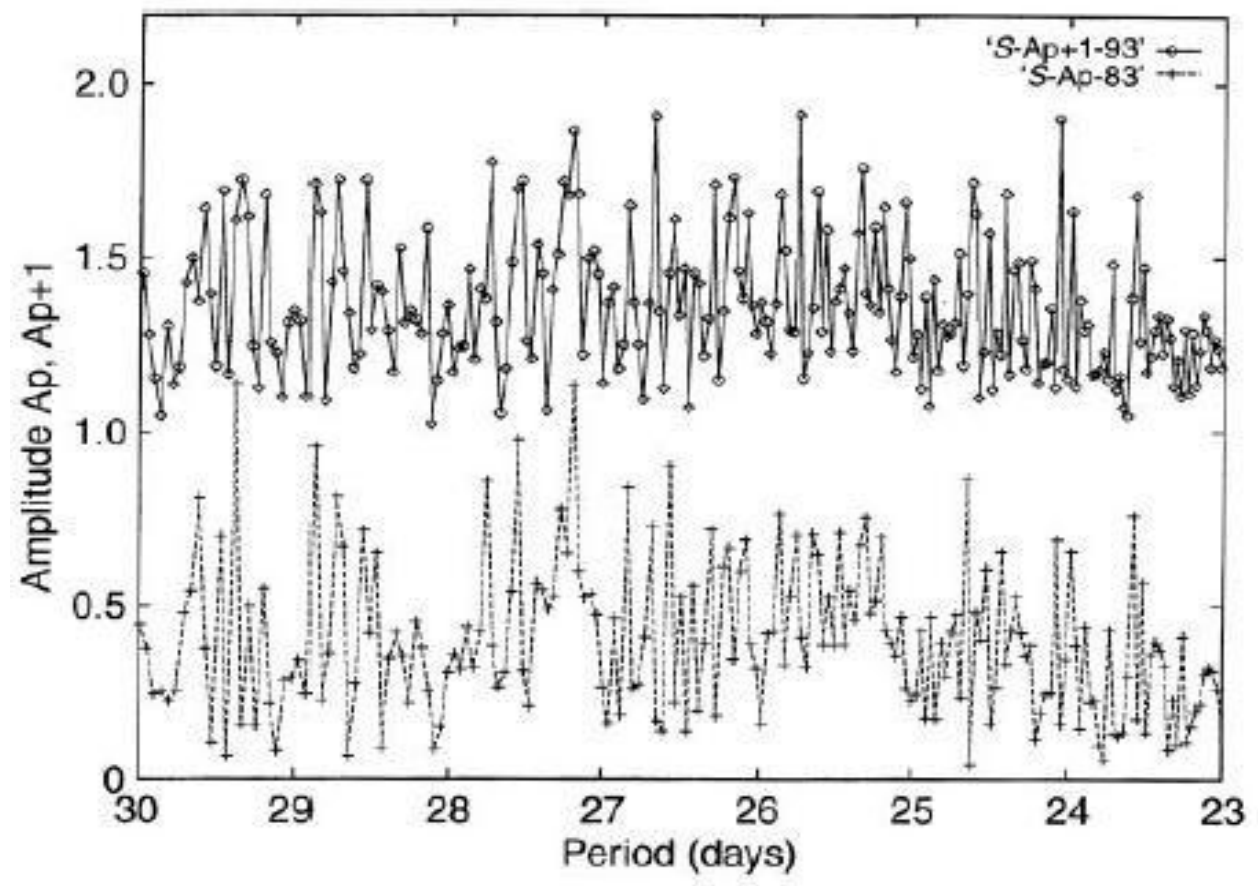

Fig. 2. Fourier spectral amplitudes for the period interval 30-23 days of the Ap time-series 1933 to 1993 (S-Ap+193) and 1933 to 1983 (S-Ap- 83), showing maximal peaks for different periodicities, depending on the length of the time-series. In the upper curve one Ap unit was added to the Fourier amplitudes S-Ap+1-93 to separate the two spectra 
No other statistically significant periods were found. The sector structure (not the activity itself) generally shows significant recurrence periods of 27 days before and during the sunspot minima, 28 to 28.5 days behind the sunspot minima, and during some 2-year intervals after the maxima. A similar solar-cycle-dependent variation of the recurrence period of the IMF region was found by (Mishra 2016) using his classification of IMF towards and away polarity based on diurnal magnetic variations at near-pole magnetic observatories.

\section{Conclusion}

In the current study our analysis of the yearly averages of Ap index and solar wind parameters $\mathrm{v}^{2}$ and Bs with the ethereal analysis of the semiannual and 28 to 28.5 - day periodicities and the diurnal UT variations of ap, give a consistent picture as the solar sources of the practical activity variations. A rough and over simplified sketch would be the following: before the solar cycle minimum, long-lived coronal holes with higher solar wind velocities extend to low solar latitudes and to the solar equator, the interplanetary magnetic field shows only slight north-south fluctuations, and the at heliospheric current sheet is near the solar equatorial plane (Mishra 2018) Therefore, geomagnetic activity is generated more by the magnitude of the solar wind velocity, and less by the southward IMF field Bs. After a solar cycle minimum, less coronal holes exist reaching the solar equator, whereas the heliospheric current-sheet and accordingly the IMF exhibit strong north-south fluctuations (Obridko 2013, Singh and Mishra (2019). This simplified view is consistent with the comprehensive description in the geomagnetic activity and its relation toward the solar sources throughout the declining phase of solar (Schreiber (1998). 


\section{References}

Arnoldy R L Signature of the interplanetary medium for substorms (1971) J Geophys Res 76:5189-5201

Berthelier A Influence of the polarity of the interplanetary magnetic field on the annual and the diurnal variations of magnetic activity (1976) J Geophys Res 81:4546-4552

Boller B R and H L Stolov (1970) Kelvin-Helmholtz instability and the semiannual variation of geomagnetic activity J Geophys Res 75:6073-6084

Cortie A L (1912) Sunspots and terrestrial magnetic phenomena 1898-1911 Mon Not R Astron Soc 73:5260

Crooker N U and E W Cliver (1994) Postmodern view of M-regions J Geophys Res 99: 23383-23390

Crooker N U and C L Sicoe (1986) The effect of the solar wind on the terrestrial environment, in Physics of the sun, vol. III, Eds. P A Sturrock, T E Holzer, D M Mihalas, and R K Ulrich, D Reidel, Dordrecht pp 193-249

Dungey J W (1961) Interplanetary magnetic fields and the auroral zones Planet Res Lett 6: 47-8

S Singh and A P Mishra (2019) Cosmic ray intensity increases during high solar activity period for the solar cycles 22 and 23, Indian J Phys 93: 139-145

Dungey J W (1994) Memories, maxims and motives J Geophys Res 99:19189-19197

Fraser-Smith A C (1972) Spectrum of the geomagnetic activity Index Ap J Geophys Res 77:4209-4220

Sham Singh, Divya Shrivastava and A P Mishra (2012) effect of solar and interplanetary disturbances on space weather Indian J Sci Res 3:121-125

Gonzalez A L C and D W Gonzalez (1987) Periodicities in the interplanetary magnetic field polarity J Geophys Res 92: 4357-4375

Gonzalez W D, A L C Gonzalez, and B T Tsurutani (1990) Dualpeak solar cycle distribution of intense geomagnetic storms Planet Space Sci 38: 181-187 1990

Gonzalez A L C, W D Gonzalez, S L G Dutra and B T Tsurutani (1993) Periodic variation in the geomagnetic activity: a study based on the Ap Index J Geophys Res 98: 9215-9231.

Green C A (1984) The semiannual variation in the magnetic activity indices Aa and Ap Planet Space Sci 32:297-305

Hirshberg J and Colburn D S (1969) The interplanetary magnetic field and geomagnetic variations united view Planet. Space Sci 17:1183-1206

Hoeksema J T, Wilcox J M and Scherrer P H (1983) The structure of the heliospheric current sheet: 1978-1982 J Geophys Res 88: 9910-9918

King J H (1977) Interplanetary medium data book, Rep. NSSCD 77-04 NASA Goddard Space Flight Cent. Greenbelt Md. 
King J H (1986) Interplanetary medium data book supplement 3, 1977-1985, Rep NSSDC 86-04, NASA Goddard Space Flight Cent. Greenbelt, $M d$

Lindsay G M, Russell C T and Luhmann J G (1995) Coronal mass ejection and stream interaction region characteristics and their potential geomagnetic effectiveness, J Geophys Res 100:16999-17013

McIntosh D H (1959) On the annual variation of magnetic disturbances Philos Trans $R$ Soc London Ser A $251: 525-552$

Murayama T(1974) Origin of the semiannual variation of geomagnetic Kp indices $J$ Geophys Res 79:297-300

Petschek H E (1964) Magnetic field annihilation, in AAS-NASA Symposium on the physics of solar flares Ed. W.N. Hess, NASA Spec. Publ. Sp-50 p. 425

Russell C T (1989) The universal time variation of geomagnetic activity J Geophys Res.Lett 16:555-558

$H$ Schreiber (1998) On the periodic variations of geomagnetic activity indices Ap and ap Ann Geophysicae 16: 510-517

Sargent H H III (1986) The 27-day recurrence index, in Solar wind-magnetosphere coupling, Eds. Y Kamide and J A Slavin,Terra Scientific Tokyo p. 143-148

$S$ Singh and A P Mishra (2015) interaction of solar plasma near-earth with reference to geomagnetic storms during maxima of solar cycle 24 Indian J Phys 89, 1227-1234

S Singh and A P Mishra (2019) Cosmic ray intensity increases during high solar activity periodfor the solar cycles 22 and 23Indian J Phys 93, 139-145

Schreiber H (1981) Correlation of geomagnetic activity indices ap with the solar wind speed and the southward interplanetary magnetic field J Geophys 49:169-175

Svalgaard L (1972) Interplanetary magnetic sector structure 1926-1971 J Geophys Res 77: 4027-4034

Svalgaard L and J M Wilcox (1975) Long-term evolution of solar sector structure, Solar Phys 41:461-475

Tsurutani B T, W D Gonzalez, A L C Gonzalez, F Tang, J K. Arballo, and M Okada, (1995) Interplanetary origin of geomagnetic activity in the declining phase of the solar cycle, J Geophys Res 100: 21717-21733

Vasilyunas V M (1997) Theoretical models of magnetic field line merging I Rev Geophys Space Phys 13: 303-336

Mishra V K, Mishra A P (2016) Study of solar activity and cosmic ray modulation during solar cycle 24 in comparison to previous solar cycle Indian J Phys 90: 1333-1339

Mishra V K, Mishra A P (2018) Long-Term Modulation of Cosmic-Ray Intensity and Correlation Analysis Using Solar and Heliospheric Parameters Sol Phys 293: 141

V N Obridko, Kh D Kanonidi, T A Mitrofanova and B D Shelting (2013) Geomagnetism and Aeronomy 53:147-156 